\title{
Localization of Rhodopsin Antibody in the Retina of the Frog
}

\author{
Maynard M. Dewey, Paul K. Davis, J. Kent Blasie and Lloyd Barr \\ Departments of Anatomy and Physiology and Biophysics, \\ Woman's Medical College of Pennsylvania, \\ Philadelphia, Pennsylvania, U.S.A. \\ and \\ Department of Physics and Biophysics Research Division, \\ Institute of Science and Technology, University of Michigan, \\ Ann Arbor, Michigan, U.S.A.
}

(Received 8 May 1968)

\begin{abstract}
A specific immune serum against frog rhodopsin has been developed in rabbits. The specificity of the antirhodopsin serum was established using the indirect Coon's antibody technique. Absorption of the serum with electrophoretically purified rhodopsin abolished its ability to stain sections of retina or isolated retinal receptor disk membranes.

Using fluorescein-labeled sheop anti-rabbit $\gamma$-globulin on sections of retina treated with antirhodopsin $\gamma$-globulin, fluorescence was observed in all retinal receptor outer segments and in the myoids and ellipsoids of retinal receptor inner segments. In addition, it was localized in cytoplasmic structures of the pigmented epithelium. Dissected retinas with intact outer segments showed fluorescence in the outer segments when the retinas were treated with antirhodopsin immune serum, washed thoroughly, incubated in fluorescein-labeled sheep anti-rabbit $\gamma$-globulin and again washed thoroughly. This is suggestive of a diffusion pathway into the outer segments. Isolated whole rod outer segments and isolated disk membranes stained uniformly by the indirect technique. From these observations we conclude that the photopigment is indeed part of the disk membranes of the outer segments and that the external membrane of the outer segment also contains photopigment. Use of the specific antirhodopsin immune serum has allowed the description of the molecular arrangement of photopigment in retinal receptor disk membranes (Blasie, Worthington \& Dewey, 1968; Blasie \& Worthington, 1969).
\end{abstract}

\section{Introduction}

This investigation was undertaken with the hope of being able to answer the following questions:

(1) Are the particles (Blasie, Dewey, Blaurock \& Worthington, 1965) which occur on the disk membranes of frog retinal receptors rhodopsin molecules?

(2) Does rhodopsin occur in the pigment epithelium and, if so, in what intracellular structures?

(3) Does rhodopsin occur in the smooth muscle cells of the iris where it might be responsible for the response of the frog iris to light, described by Barr \& Alpern (1963)? 
In this paper we describe the development and specificity of antisera produced in rabbits against rhodopsin isolated from frog retina. Further, we describe the immunohistochemical localization of this specific antiserum in the retina of the frog.

Experimental answers to the first two questions were obtained and are discussed in this paper and the succeeding two papers (Blasie, Worthington \& Dewey, 1969; Blasie \& Worthington, 1969). A satisfactory answer to the third question was not obtained due to the high degree of autofluorescence of the frog iris. This made impossible an evaluation of the specificity of staining with the antiserum in the iris. This difficulty can be overcome by using ferritin or peroxidase-labeled $\gamma$-globulin and electron microscopy. Such studies are now in progress.

\section{Materials and Methods}

\section{(a) Preparation of rhodopsin}

Rhodopsin was prepared by a variety of methods, all of which were modifications of one used by Wald \& Brown (1952) for cattle rhodopsin. In the method finally adopted, retinas of 12 dozen small, 24-hr dark-adapted frogs (Rana pipiens obtained from Steinhilber \& Sons, Oshkosh, Wisc.) were removed and suspended in $16 \mathrm{ml}, 0.1 \mathrm{~m}$-phosphate buffer, pH 6.5 , containing $40 \%(\mathrm{w} / \mathrm{v})$ sucrose. They were disrupted by means of a large syringe and 16 gauge needle. The suspension was divided equally between 2 cellulose nitrate centrifuge tubes. Four ml. of buffer (0.10 M-phosphate, $\mathrm{pH} 6 \cdot 5)$ were then carefully layered above the suspension and the tubes centrifuged in a Spinco model L no. 40 rotor for 15 $\mathrm{min}$ at $20,000 \mathrm{rev} . / \mathrm{min}$.

A clean syringe was used to transfer the rod outer segments which had collected at and immediately below the buffer-sucrose interface to a new, cooled tube. Buffer was then added to the original tubes, and the mixture stirred and centrifuged $(20,000 \mathrm{rev} . / \mathrm{min}$ for $10 \mathrm{~min}$ ). After discarding the supernatant fraction, the residue was disrupted again with sucrose solution and the buffer-layering repeated. This was repeated 4 times and the outer segments collocted from the 4 flotations woro combined.

The outer segments were then sedimented by centrifugation following the addition of buffer, washed with buffer and finally distilled water. They were stirred, first with $40 \%$ (w/v) alum-water (1/2 hr), and then with $4 \%$ neutralized formalin (15 min). A final buffer wash was made before extractions.

Four extractions, each with $4 \mathrm{ml}$. of $2 \%$ (w/v) digitonin-0.1 M-phosphate buffer, pH 6.5, were carried out in the refrigerator, with occasional stirring for 1 to $3 \mathrm{hr}$. The resulting solutions were cleared of debris and precipitated digitonin by centrifugation and frozen with liquid nitrogen for storage.

Variations involved exclusion of the formalin treatment, and/or inclusion of 3 petroleum ether (low boiling, Baker's reagent) washes of the sedimented and dried outer segments. Drying was accomplished by mixing with anhydrous sodium sulfate. After the washes, the plug was broken up, spread over the surface of a microscope slide, and left for about $1 \mathrm{hr}$ during which the petroleum ether evaporated. Buffer washes were then necessary to remove the sodium sulfate. Rhodopsin from the dried plug was then extracted in digitoninphosphate solution. None of these variations gave as satisfactory results as the procedure described above.

If the rhodopsin preparation showed considerable contamination, as frequently occurred during winter months, the purity of the digitonin-rhodopsin solutions were improved by passage through a Sephadex G200 column, 5 in. long and 1 in. in diam. Buffer $(0 \cdot 1 \mathrm{M}-$ phosphate, pH 6.5) was used to prepare the slurry, and 1\% digitonin in the buffer to wash the rhodopsin through the column. Small amounts of rhodopsin were also purified and concentrated by electrophoresis in cellulose acetate.

\section{(i) Spectroscopy}

\section{(b) Methods and criteria of assay of rhodopsin preparations}

Digitonin-rhodopsin solutions were cleared by centrifugation and spectra were obtained with $1 \mathrm{ml}$. Pyrocell cuvettes (path length, $1 \mathrm{~cm}$ ) and a Gilford model 2000 spectrophoto- 
meter using a Beckman DU monochromator. Calibration was accomplished with Gilford standard filters, and the wavelength calibrated with a mercury lamp. Since accuracy of $\pm 1 / 2 \%$ was achieved up to $3 \cdot 0$ absorbance units, dilutions were seldom necessary. A water blank was used and $0.6 \%$ digitonin served as a control. Hubbard (1954) has shown that for cattle rhodopsin preparations, the digitonin concentration stabilizes at about $6 \mathrm{mg} / \mathrm{ml}$./ unit absorbance at $500 \mathrm{~m} \mu$; the remainder of the digitonin precipitates. In the event of digitonin precipitation, as evidenced by clouding of the solution and abnormally high absorbance values in the visible region, the sample and/or control were recentrifuged. Corrections of the spectrum could then be made by subtracting the digitonin control.

\section{(ii) Nitrogen analysis}

Given a constant concentration of rhodopsin, with its characteristic maximum absorption at $500 \mathrm{~m} \mu$, the nitrogen content would be reduced as protein impurities are eliminated. Thus, the index $\mathrm{N} / A$ (defined as (concn $\mathrm{N}$ in $\mathrm{mg} / \mathrm{ml}$.) $/ A_{500}$ ) should decrease with increasing purity. Nitrogen content of $0.25 \mathrm{ml}$. samples was analysed by a microKjeldahl method. It was found that $30 \mathrm{~min}$ were sufficient for digestion.

\section{(iii) Electrophoresis in cellulose polyacetate}

Electrophoresis was performed on Gelman Sepraphore strips (cellulose polyacetate) under a variety of conditions: 7 to $20 \mathrm{v} / \mathrm{cm}$, durations of 1 to $3 \mathrm{hr}$, samples of 3 to $25 \mu \mathrm{l}$. of the digitonin-rhodopsin solution, and range of $\mathrm{pH}$ from 4 to 9 . The buffers used were 0.05 $\mathrm{m}$-acetate, Tris, Barbital, or phosphate; the latter two proved to be the most useful. Strips were stained with $0.2 \%$ Ponceau $R$ in $5 \%$ trichloroacetic acid and $0.001 \%$ Nigrosin in $2 \%$ acetic acid.

When electrophoresis was done in the refrigerator for $2 \mathrm{hr}$ at $20 \mathrm{v} / \mathrm{cm}$, the components were sufficiently separated to allow cutting of individual bands from the strip. Rhodopsin remained unbleached and was identifiable as a red band. Samples of $25 \mu \mathrm{l}$. were applied. and separated in this way, thus providing a method for concentrating a very pure rhodopsin.

\section{Preparation of antisera}

(c) Development and evaluation of antisera

Each of 6 large rabbits received $5 \mathrm{ml}$. of antigen mixture (1:1 mixture of rhodopsin preparation and complete Freund's adjuvant) subcutaneously, in footpads, cervical and scapular regions. Rabbits 3 and 4 were injected only after the mixture had been exposed to light, but $1,2,5$ and 6 received what is thought to have been unbleached rhodopsin. After injections were made, the syringe drippings were still red. Usually, the injections contained about $2.5 \mathrm{mg}$ of rhodopsin.

\section{(i) Frozen sections}

\section{(d) Immunohistochemistry}

Eyes were removed from spinally-pithed frogs, frozen in egg albumin by submersion in an isopentane-dry ice mixture and then transferred to liquid nitrogen for storage. Sections $\left(6\right.$ to $10 \mu$ ) were obtained in an International Harris Cryostat, washed in $\mathrm{PO}_{4}$ buffered

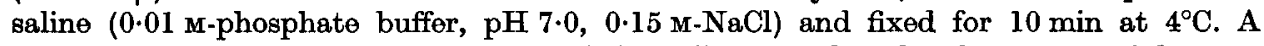
number of fixatives were tried but $10 \%$ formalin proved to be the most satisfactory. Following fixation the sections were washed with $\mathrm{PO}_{4}-$ saline, treated with antiserum for 30 min, washed with $\mathrm{PO}_{4}$-saline and finally treated with fluorescein-labeled sheep antirabbit $\gamma$-globulin for $30 \mathrm{~min}$. The sections were then washed in $\mathrm{PO}_{4}$-saline and mounted.

Fluorescein-labeled sheep anti-rabbit $\gamma$-globulin was prepared for us by Drs G. B. Pierce and R. Midgely. The fluorescein-labeled $\gamma$-globulin was always absorbed against homogenized frog livers and eye bulbs. Control sections treated with this reagent alone showed no staining.

Immunohistochemistry was also performed on isolated retinal receptor outer segments and disk membranes. Outer segments were obtained by gently shaking a dark-adapted retina in Ringers solution and collecting drops of the solution on the surface of Formvarcoated copper grids. Disk membranes were obtained by disrupting dark-adapted retinas in $45 \%$ sucrose with a glass syringe, floating disrupted outer segments on a $45 \%$ sucrose- 
$0.1 \mathrm{~m}$-phosphate buffer (pH 7.4) interface at $25,000 \mathrm{rev} . / \mathrm{min}$ for $15 \mathrm{~min}$, and subsequently collecting the disk membranes from the interface on Formvar-coated copper grids. Purity of such preparations was established by electron microscopy (see Blasie et al., 1969). The grids with specimens were partially dried ( 1 to $2 \mathrm{sec}$ ), washed with $\mathrm{PO}_{4}$-saline and steined using the same procedures as described for the frozen sections. The grids could then be viewed with the light microscope. In this way we were able to check rapidly the specificity of antisera and observe results free from the effects of freezing, fixation and background staining.

(ii) Electrophoresis

The immunochemistry was also accomplished directly onto cellulose acetate electrophoresis strips. Here, however, it was necessary to wash very vigorously for $10 \mathrm{~min}$ in order to reduce non-specific effects. Both fluorescein and ${ }^{131} \mathrm{I}$-labeled anti-rabbit $\gamma$-globulin (kindly supplied to us by Dr Midgely) were used. In the former case a source of ultraviolet light was directed on the strips to excite fluorescence. With the latter, readings were made with a liquid-scintillation counter for times corresponding to a statistical counting error less than $10 \%$.

\section{Results}

Maxima occur in the absorption spectrum of rhodopsin at $280 \mathrm{~m} \mu$ and $500 \mathrm{~m} \mu$, a minimum at $400 \mathrm{~m} \mu$ (Fig. 1). Purity has been associated with a minimization of the ratios of two spectral indices: $P_{280}$ and $P_{400}(P \lambda$ is defined as (absorbance at $\lambda)$ ) (absorbance at $500 \mathrm{~m} \mu$ )). Wald (1938) has estimated that pure rhodopsin cannot have a $P_{400}$ less than 0.20. A $P_{280}$ of 2.05 was reported for cattle rhodopsin (Collins, Love \& Morton, 1952; Hubbard, 1954). Since proteins may be present without affecting the $P_{400}$, the $P_{280}$ is the more accurate index of protein contamination. A useful concentration index for the chromophore is simply the $A_{500}$, as the absorbance there is due almost entirely to rhodopsin.

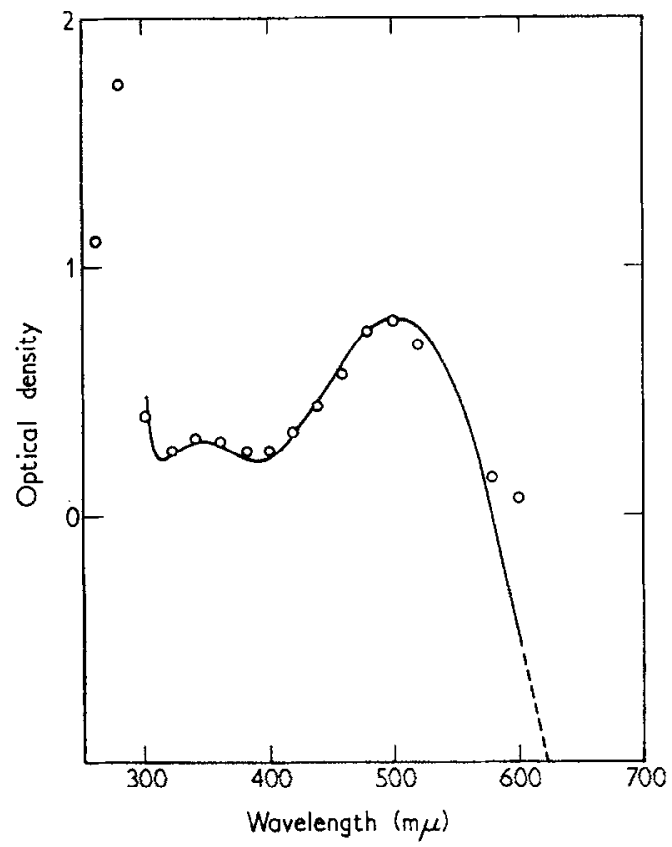

Fia. 1. Absorption spectrum of rhodopain. (Curve taken from Wald, 1949.)

Circles from average preparation of rhodopsin used in these experiments. Such a preparation would show two impurity fractions upon electrophoresis. 


\section{(a) Purity and concentration of preparations}

The basic procedure for preparing rhodopsin described was found to yield about $12 \mathrm{ml}$. of solutions with typical $P_{400}=0.3 \pm 0.1$ and $P_{280}=2 \cdot 5 \pm 0.3$. Twelve dozen frogs were usually sufficient to assure one extract with $A_{500}$ of $1 \cdot 0$ to $2 \cdot 0$ and three more with $A_{500}$ vaules of 0.6 to $\mathbf{1 . 2}$.

Made without a formalin treatment, some 20 preparations were found to have typical $P_{400}$ and $P_{280}$ values of $0 \cdot 3 \pm 0 \cdot 1$, and $2 \cdot 4$ to $3 \cdot 2$, respectively. Use of formalin appeared to assure a $P_{280}$ of about $2 \cdot 5$. On two occasions we obtained $P_{400}$ values of $0 \cdot 20 \pm 0.01$, and several times, $P_{280}$ values of $2 \cdot 25 \pm 0 \cdot 05$. As we did not determine the $P_{280}$ for a number of our better preparations, we cannot describe $2 \cdot 25$ as the minimum value obtainable.

Though our best preparations were achieved without the petroleum ether treatment, its use does appear to increase the likelihood of $P_{400}$ values less than $0 \cdot 30$. The pre-drying is essential.

Sephadex column separation served as a method by which exceptionally contaminated preparations $\left(P_{400}>0 \cdot 6, P_{280}>4\right)$ could be improved to normal levels of purity $\left(P_{400} \sim 0.35, P_{280} \sim 3\right.$ ). With Sephadex G200, resolution is limited to molecular weights below 270,000 . Since some of the proteins, including rhodopsin, are bound in large micelles with molecular weights in excess of this, it is the lipid contamination which is chiefly reduced and thus it is not surprising that the major spectral improvement is in the visible region.

\section{(b) Correlation of electrophoretic results with spectral and nitrogen indices}

Data from nitrogen analysis indicate that electrophoresis at $\mathrm{pH} 7.5$ (phosphate buffer) and 8.0 (Barbital buffer) reflect protein purity in reasonably reliable fashion.

Figure 2 includes sketches of typical electrophoretic patterns at $\mathrm{pH}$ values $\mathbf{7 \cdot 5}$ and $8 \cdot 0$, and Table 1 lists the various indices for such preparations.
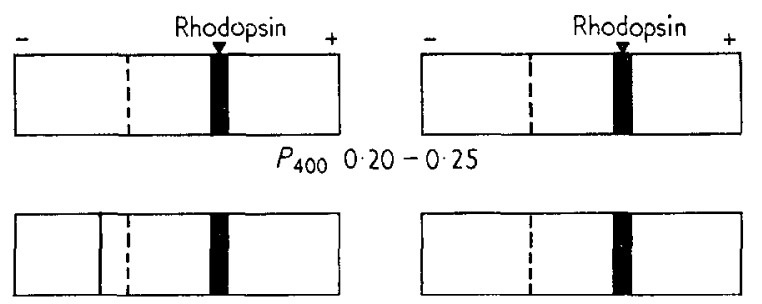

$P_{400} 0.28-0.33$
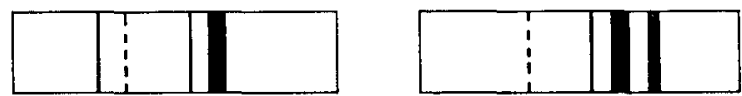

$P_{400} 0 \cdot 35-$

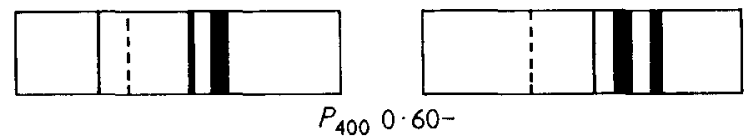

$\rightarrow 0.05 \mathrm{M}$ - Phosphate, $\mathrm{pH} 7.5-\quad-0.05 \mathrm{M}$-Borbital, $\mathrm{pH} 8.0-$

FIG. 2. Summary of drawings of cellulose acetate strips following electrophoresis of rhodopsin preparations of varying purities. 
TABLE 1

Rhodopsin preparations

\begin{tabular}{|c|c|c|c|c|}
\hline Sample no. & $P_{\mathbf{4 0 0}}$ & $P_{280}$ & $\begin{array}{c}\text { No. of } \\
\text { bends in } \\
\text { electrophoresis }\end{array}$ & $\begin{array}{c}\text { N conen } \\
(\mathrm{mg} / \mathrm{ml} .) \\
\left.A_{\mathrm{so0}}\right)\end{array}$ \\
\hline $\mathrm{K} 10 \mathrm{~A}$ & 0.20 & & 1 strong & \\
\hline 1 & $0 \cdot 28$ & & $\begin{array}{l}1 \text { strong } \\
1 \text { very weak }\end{array}$ & $0 \cdot 16$ \\
\hline 7 & 0.33 & & $\begin{array}{l}1 \text { strong } \\
1 \text { very weak }\end{array}$ & $0 \cdot 16$ \\
\hline 35 & $0 \cdot 33$ & $2.25 \pm 0.05$ & $\begin{array}{l}1 \text { strong } \\
1 \text { very weak }\end{array}$ & 0.22 \\
\hline 40 & $0 \cdot 35$ & $2 \cdot 4 \pm 0 \cdot 1$ & $\begin{array}{l}1 \text { strong } \\
1 \text { medium } \\
1 \text { very weak }\end{array}$ & 0.27 \\
\hline 34 & $0 \cdot 6$ & $2 \cdot 8$ & $\begin{array}{l}2 \text { strong } \\
1 \text { very weak }\end{array}$ & 0.31 \\
\hline
\end{tabular}

The particular $\mathrm{pH}$ values and buffers chosen seemed to result in maximum resolution and sharp patterns without bleaching the rhodopsin. Results of phosphate runs (pH 7.5) tended to be sharper and darker, but in Barbital the separations were faster.

Even preparations with an $\mathrm{N} / A$ index of 0.16 gave one impurity line, but this was so weak as to be visible only in phosphate when stained with nigrosin. As the N/A index increased, this impurity line darkened and a second line appeared whose apparent density equalled that of the rhodopsin band as the N/ $A$ index approached $0 \cdot 30$. Thus, it would appear that the electrophoretic methods separated protein impurities from rhodopsin.

It is interesting to note that the $P_{280}$ and $N / A$ index appeared to correlate only semi-quantitatively.

\section{(i) Immunohistochemistry}

\section{(c) Specificity of antisera}

Antisera against both bleached and unbleached rhodopsin were always absorbed against fresh homogenized frogs lacking eye bulbs, followed by absorption with activated charcoal, and were usually tested for their ability to stain a number of tissues and organs from the frog. Antisera so absorbed did not stain structures in sections of skeletal muscle, cartilage, kidney, liver, spleen or brain.

Sections of the retina treated with immune antirhodopsin serum showed localized staining in the outer segments and possibly in the ellipsoids and myoids of the receptor cells and in the pigment epithelium of the retina (Plate $I(a)$ and (b)). Of the outer segments, rods and cones stained with equal intensity. 


\section{(ii) Reactions of electrophoretic fractions of rhodopsin with immune serum}

Cellulose acetate strips containing electrophoretic fractions of rhodopsin preparations were treated with absorbed immune serum, washed thoroughly and treated with either fluorescein or ${ }^{131}$ I-labeled sheep anti-rabbit $\gamma$-globulin. The rhodopsin fraction was identified as a red band which bleached upon exposure to light. Absorbed immune serum reacted only with the rhodopsin fractions as shown by the localization of the fluorescence due to fluorescein-labeled sheep anti-rabbit $\gamma$-globulin or the radioactivity due to ${ }^{131} \mathrm{I}$-labeled sheep anti-rabbit $\gamma$-globulin.

\section{(iii) Absorption of immune serum with electrophoretically isolated rhodopsin}

Rhodopsin preparations with $P_{400}$ values of $0 \cdot 20$ to 0.25 were electrophoretically separated on cellulose acetate using phosphate buffer at $\mathrm{pH} 7 \cdot 5$. Rhodopsin fractions identified by their red color were cut from the strips and pooled. Immune serum which had proved satisfactory using the critera described above were absorbed against these isolated rhodopsin fractions. Controls were run by absorbing the same sera against either clean cellulose acetate strips, impurity fractions from rhodopsin preparations with $P_{400}$ values higher than $0 \cdot 60$ or protein fractions of rabbit serum isolated on cellulose acetate strips. Plate $I(b)$ and (c) illustrates such an experiment. Absorption against the rhodopsin fractions abolished staining in the retina by the immune rabbit antirhodopsin serum. Absorption against clean cellulose acetate, impurity fractions of rhodopsin or rabbit serum proteins did not affect the fluorescence obtained with the immune serum followed by fluorescein labeled anti-rabbit $\gamma$ globulin. These tests, we feel, establish the specificity of the immune serum used in subsequent experiments. All subsequent experiments used this specific immune rabbit antirhodopsin serum.

\section{(d) Distribution of staining in sections of retina}

\section{(i) Receptor cells}

Sections stained with immune serum followed by fluorescein-labeled sheep antirabbit $\gamma$-globulin showed bright green fluorescence in all outer segments throughout the retina. The fluorescence appeared nearly uniform along a single outer segment. No striations were apparent. No distinction in staining could be made between red and green rods or single and double cone outer segments. Specific fluorescence was observed in the myoids and ellipsoids of all inner segments. This staining was less intense than that observed in the outer segments.

\section{(ii) Pigment epithelium}

In light-adapted eyes in which pigment migration into epithelial processes between the outer segments had occurred, specific fluorescence was apparent in the scleral side of the pigment epithelium (Plate I(b) and Plate II(b)). Staining probably occurred in the vitreal side of the epithelium except it was obscured visually by the abundant pigment. The observable fluorescence in the epithelium occurred diffusely and also in discrete, focal patches. These fluorescent punctate bodies appeared similar in size and distribution to the structures which stain in these cells with Baker's hematoxylin reagent (Lillie, 1965) for the demonstration of phospholipids (Plate II(a)).

The more diffuse staining corresponds to the network throughout the cytoplasm of these cells which is also delineated by the Baker method for demonstrating phospholipids (Plate II(a)). 


\section{(iii) Staining of intact outer segments}

Retines were carefully dissected from eye bulbs and maintained in Ringer's solution. Integrity of the retina and outer segments was checked by observation with bright field microscopy (Plate III(a)). Retinas were then covered with immune serum diluted with $\mathrm{PO}_{4}$-saline for 30 minutes, washed with several rinses of Ringer's solution, and treated with fluorescein-labeled sheep anti-rabbit $\gamma$-globulin for 30 minutes. Retinas were then thoroughly washed with Ringer's solution. Intact outer segments fluoresced following such a treatment (Plate III(a) and (b)). Control retinas treated with normal rabbit serum and the fluorescein-labeled reagent were negative (Plate III(c)).

Intact outer segments stained in this manner did not show cross-striations. When viewed end-on they appeared brighter than when observed from the side. Further, when viewed end-on they fluoresced uniformly. There was no prominent peripheral ring of fluorescence. While these observations are in no way conclusive they suggest that $\gamma$-globulins were able to diffuse along an extracellular pathway to the disk membranes in the outer segment and, therefore, that antigenic sites of photopigments occur on the external membrane of the outer segments. The only alternative seems to be that the $\gamma$-globulin is able to pass across the external membrane in these preparations.

\section{(iv) Staining of outer isolated segments and disk membranes}

Both isolated outer segments and disk membranes collected on formvar-coated copper grids fluoresced brilliantly following treatment with specific antirhodopsin serum and fluorescein-labeled sheep anti-rabbit $\gamma$-globulin (Plate IV(b) and (d)). Control preparations treated with normal rabbit serum were negative (Plate IV(a) and (c)). Isolated disk membranes appear to be a mixture of individual lobes or whole disk membranes from rod outer segments. From fluorescent microscopic observations these disk membranes stained evenly across their surfaces. Individual isolated outer segments also stained evenly showing no cross striations or differential staining along the surface of the outer segment.

\section{Discussion}

The development of immune serum which reacts specifically with an electrophoretically purified rhodopsin hus led to the molecular localization of rhodopsin in retinal receptor outer segment disk membranes (Blasie et al., 1969; Blasie \& Worthington, 1969).

Use of the antiserum in immunohistochemical procedures applied to sections of the retina and to isolated, intact retina and isolated outer segments, may lead to the solution of additional questions relative to the morphology and functional interrelationships of retinal receptor outer segments, pigment epithelium and photopigment localization in photosensitive cells of the iris.

\section{(a) Specificity of the immune serum}

Of the three procedures used to establish the specificity of our immune serum, the immunohistochemical method appeared to be the most satisfactory and critical. Absorption of the immune serum with electrophoretic fractions of rhodopsin isolated 
Plate I. (a) A light micrograph of portion of retina of the frog. The eye was light-adapted prior to fixation and pigment of pigmented epithelium has migrated between outer segments as far as the external limiting membrane. Some pigment remains near vitreal side of nuclei of pigmented epithelial cells. Formalin-fixed. Hematoxylin and eosin. $(\times 300)$.

(b) A section of light-adapted retina treated with immune antirhodopsin serum followed by fluorescein-labeled sheep anti-rabbit $\gamma$-globulin. Fluorescence is seen in the pigmented epithelium, retinal receptor outer segments and in the ellipsoids and myoids of the inner segments except where it is obseured by pigment. Other regions of retinu and tissues of peripheral portions of eye bulb as well as socket are negative (compare with control (d)). Artifacts over inner nuelear layer indicate level of positive fluorescence. Formalin-fixed $(\times 300)$.

(c) Adjacent section to that illustrated in (b) treated with immune antirhodopsin serum absorbed against electrophoretic fractions of rhodopsin followed by fluorescein-labeled sheep anti-rabbit $\gamma$-globulin.

Absorption of antirhodopsin immune serum against pooled electrophoretic fractions of rhodopsin abolishes all staining in the retina. Positive fluorescence is indicated by artifacts while background fluorescence is comparable to control (d). Formalin-fixed $(\times 300)$.

(d) Adjacent section to that illustrated in (c) treated with non-immune rabbit serum followed by fluorescein-labeled sheep anti-rabbit $\gamma$-globulin. Positive fluorescence is indicated by artifacts. Formalin-fixed $(\times 300)$.

PLATE II (a) Section of dark-adapted retina showing pigmented epithelium stained by Baker acid hematin procedure for the demonstration of phospholipids. Right-hand arrow indicates diffuse cytoplasmic network which gives a positive reaction with this method. Left-hand arrow indicates punctate bodies which are probably myeloid bodies $(\times 430)$.

(b) Section of dark-adapted retina showing pigmented epithelium treated with immune antirhodopsin gerum followed by fluorescein-labeled sheep anti-rabbit $\gamma$-globulin. Left-hand arrow indicates diffuse cytoplasmic fluorescing organelles. Right-hand arrow indicates punctate fluorescing organelles. These correspond to structures stained by Baker's acid hematin reagent (a). Formalin-fixed $(\times 500)$.

Plate III. (a) Bright-field photomicrograph of dissected retina showing end-on view of outer segments. Such dissection leaves outer segments visually unaltered $(\times \mathbf{3 0 0})$.

(b) A retina dissected as shown in (a), treated with immune antirhodopsin serum, washed thoroughly, treated with fluorescein-labeled anti-rabbit $\gamma$-globulin and washed thoroughly. The photograph was taken so as to show side-on and partial end-on views of outer segments. Note uniform fluorescence throughout outer segment $(\times 300)$.

(c) A retina treated identically to that illustrated in (b) except normal rabbit serum replaced the antirhodopsin serum. Outer segments are negative; positive fluorescence is indicated by artifact in center of field $(\times 300)$.

Plate IV. (a) An isolated outer segment collected on electron microscope grid treated with normal rabbit serum followed by fluorescein-labeled sheep anti-rabbit $\gamma$-globulin. Outer segment has not stained; positive fluorescence indicated by artifact in upper portion of field $(\times 500)$.

(b) An isolated outer segment similar to that shown in (a) but treated with immune antirhodopsin serum prior to fluorescein-labeled sheep anti-rabbit $\gamma$-globulin. Note uniformity of fluorescence of outer segment. Dark region in its center is region underlying bar of grid $(\times 500)$.

(c) Isolated retinal receptor disk membranes on electron microscope grid treated with norma] rabbit serum followed by fluorescein-labeled sheep anti-rabbit $\gamma$-globulin. Disk membranes do not show positive fluorescence $(\times 500)$.

(d) Isolated disk membranes similar to those shown in (c) but treated with immune antirhodopsin serum prior to fluorescein-labeled sheep anti-rabbit $\gamma$-globulin. Disk membranes fluoresce brilliantly and uniformly across their surface. For electron micrograph of similar preparation showing organization of membranes see the following paper (Blasie et al., 1969, Plate I) $(\times 500)$. 


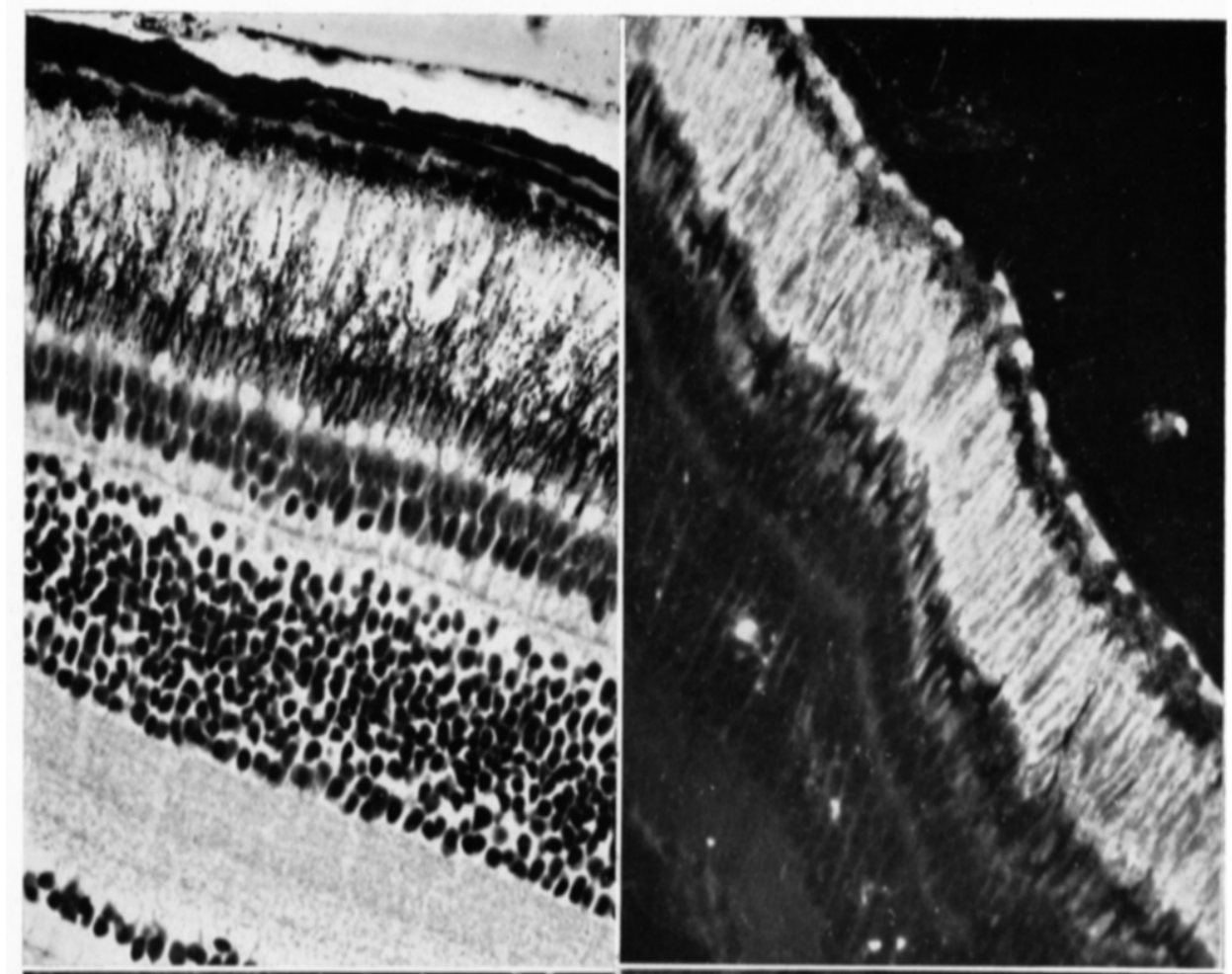

(b)

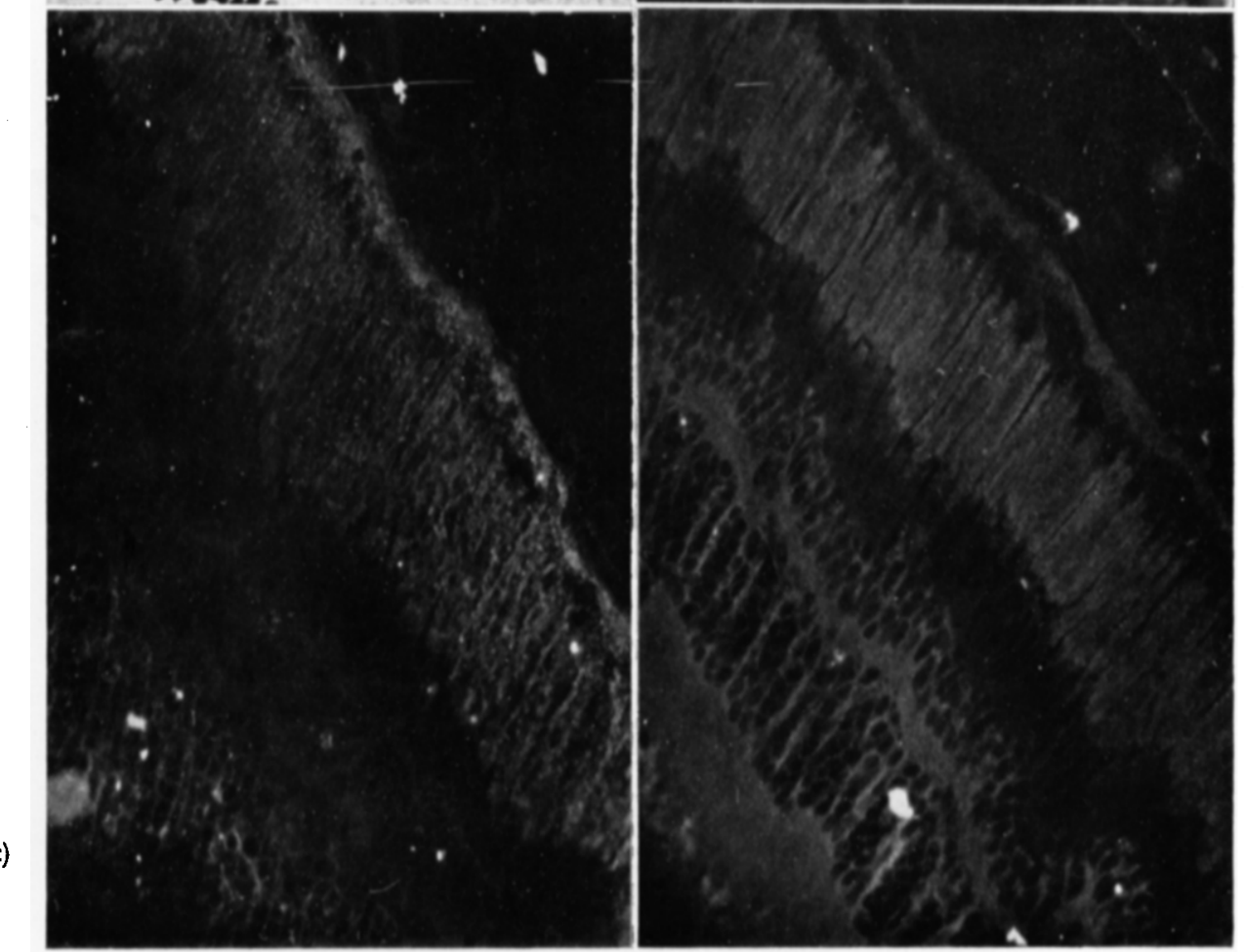

(d) 


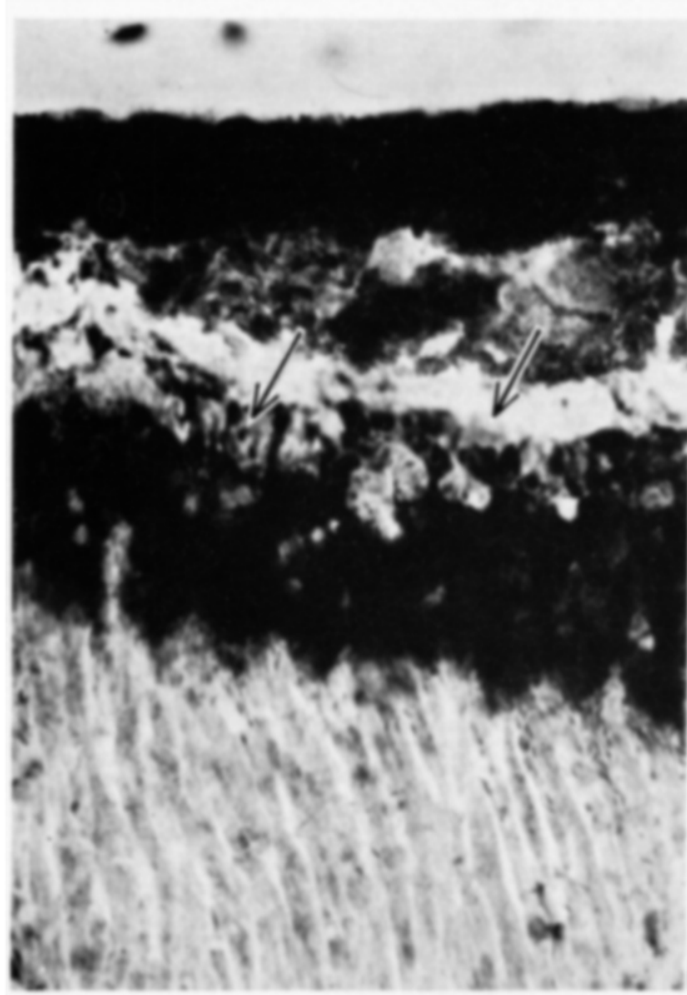

(a)

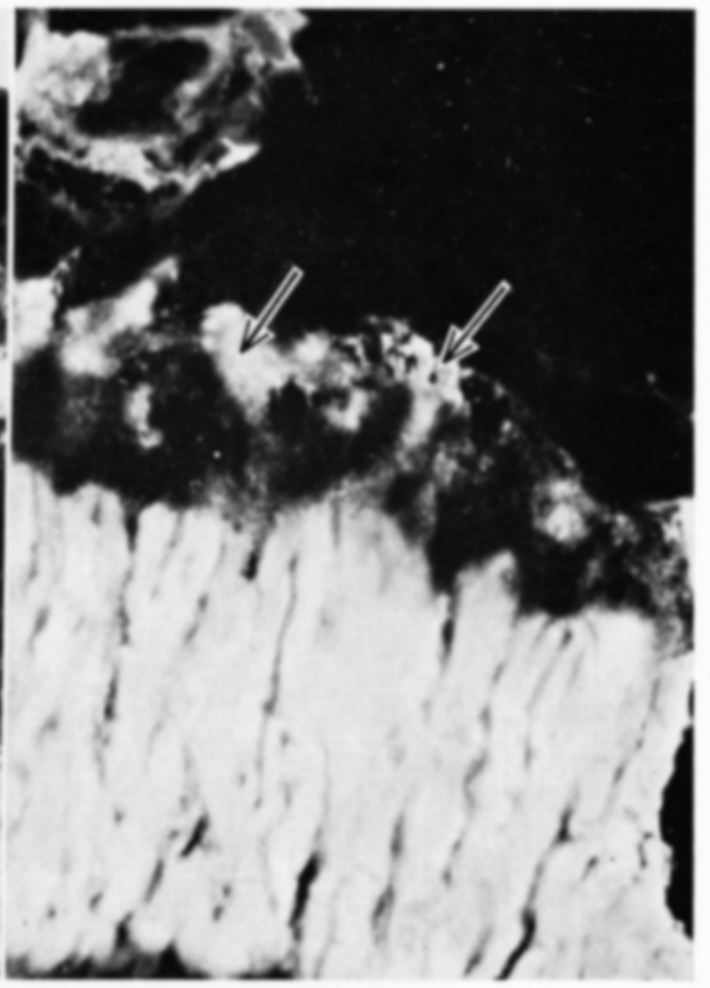

(b)

Plate II 


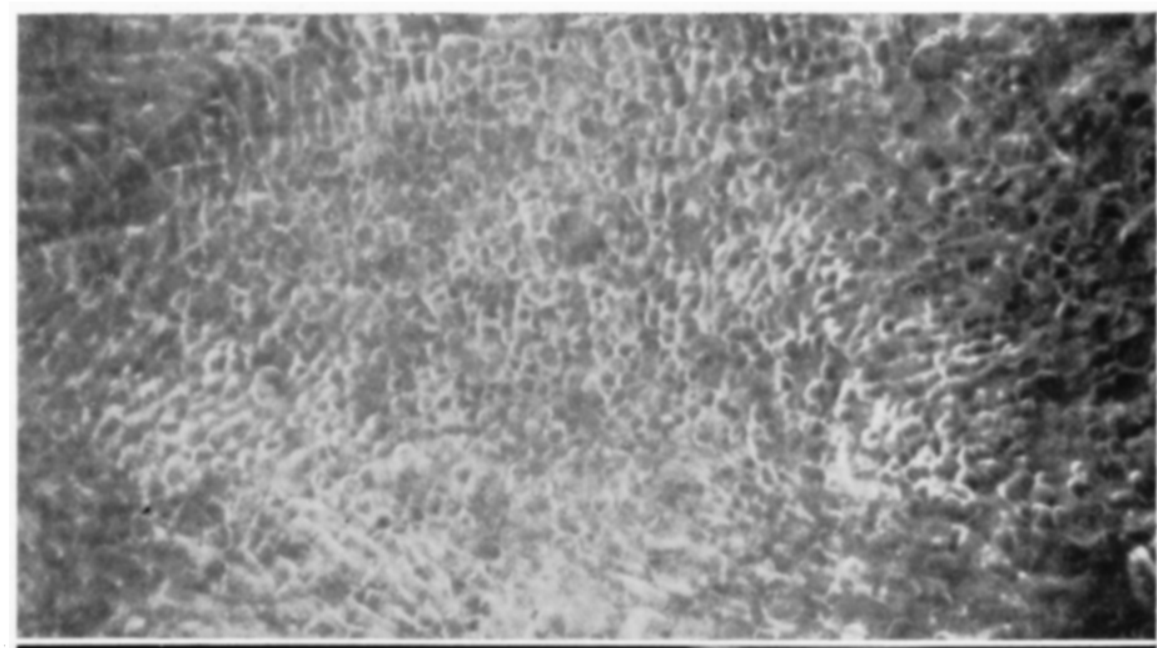

(a)

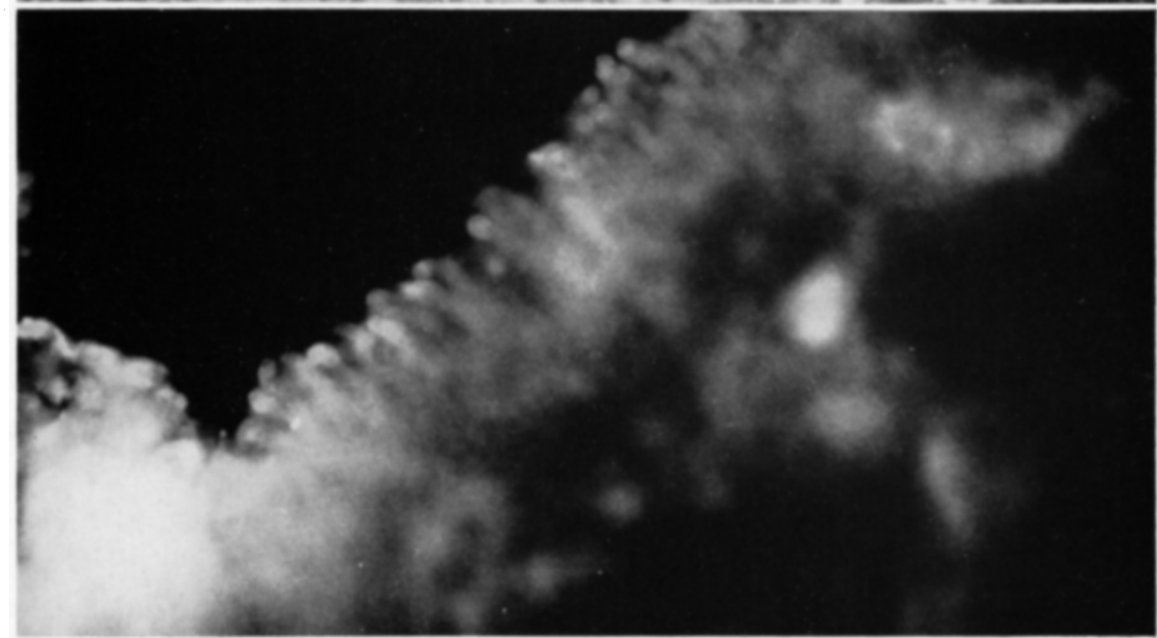

(b)

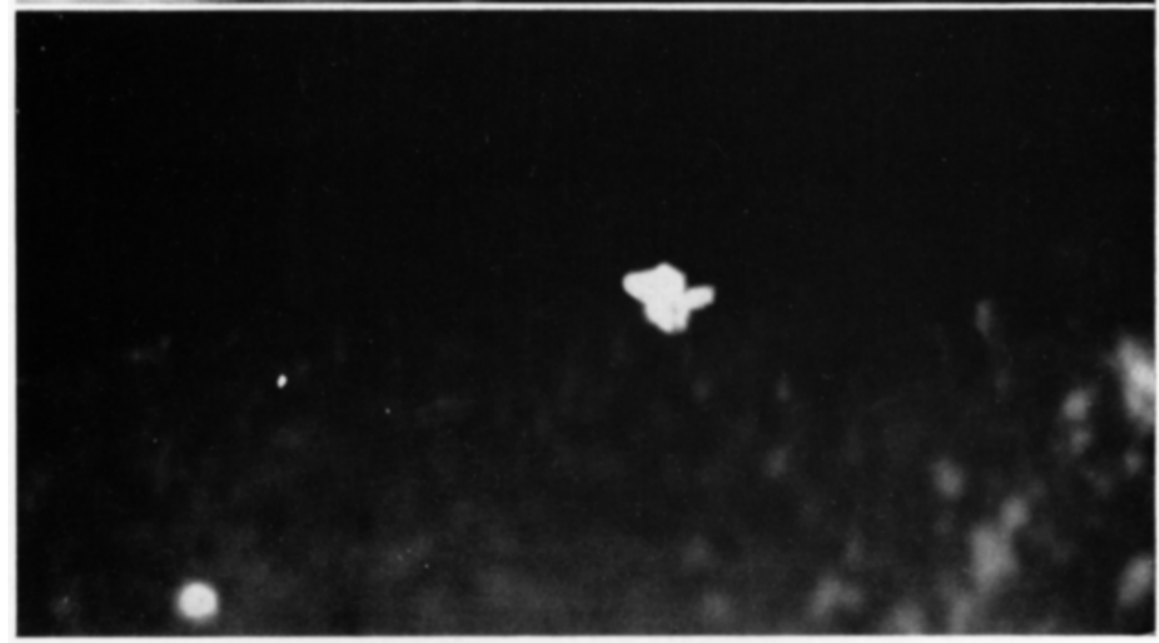

(c)

Plate III 


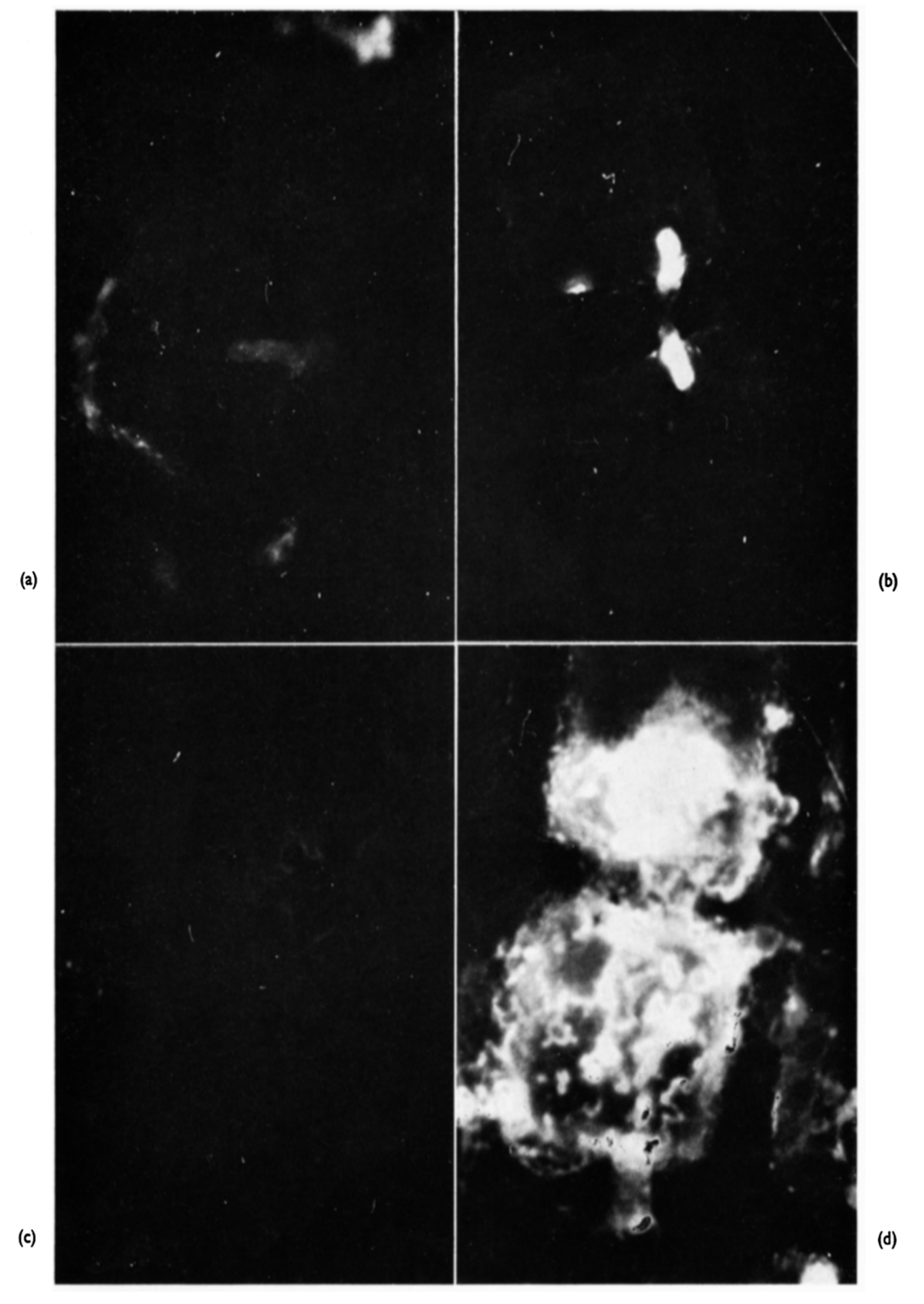

Plate IV 
from rhodopsin preparations with $P_{400}$ values of 0.20 to $0 \cdot 25$ abolished all staining by the immune sera of sections of retinas and isolated disk membranes. From spectral, nitrogen and electrophoretic analysis such rhodopsin preparations have minimal protein contaminants. Conversely impurity fractions isolated from rhodopsin preparations with $P_{400}$ values greater than $0 \cdot 60$ did not extinguish specific staining by the immune serum. It seems probable that the antigenic sites are associated with the opsin moiety of rhodopsin since electrophoretic fractions of completely bleached rhodopsin preparations stained with the antiserum.

\section{(b) Cross-reactivity of immune serum with other photopigments}

Since the immune sera used in these experiments stained all outer segments in the retina of the frog, there must be cross-reactions between the photopigments of retinal receptors. The frog retina contains two types of rods, red and green, which constitute 55 and $11 \%$ of the outer segments, respectively, in the central portions of the retina, and two types of cones, single and double, which comprise the remaining $33 \%$ of outer segments. In the peripheral portion of the retina the cone population is somewhat lower, $20 \%$ (for review see Nilsson, 1964b). It is possible that our rhodopsin preparations used as antigens were contaminated by other opsins. If various opsins have similar electrophoretic mobilities our methods would not distinguish between them. However, judging from spectral criteria and nitrogen analysis this possibility appears remote.

\section{(c) Immunohistochemistry of retinal receptors}

This work confirms the long held view that photopigment molecules are part of the disk membranes of retinal receptor outer segments. Further, it establishes that the photopigment molecules occur sufficiently near the surface of these membranes so that their antigenic regions are exposed. Observation of the distribution of fluorescence following treatment of isolated disk membranes by the indirect method also confirms the homogeneity of distribution of photopigment molecules along the surface of disk membranes. Thus, the assumption of homogeneity of the photopigment distribution along the disk in making estimates of number of photopigment molecules/disk, using optical densities of outer segments and numbers of disks/outer segment seems safe.

Our studies are also strongly suggestive that photopigment occurs on the external membrane of the retinal receptor outer segment. Operationally, this might serve to increase the efficiency of scattered photon absorption by the outer segments particularly since it lies in a plane normal to that of the disk membranes themselves.

Localization of the antirhodopsin antigen in the myoid and ellipsoid of retinal receptors raises a question as to its biological role. Whether this represents only opsin localization or opsin plus retinene could not be distinguished by our procedures. Further, the precise intracellular distribution could not be determined in our preparations. Young (1967) has shown using tritiated methionine and radioautography, that in the frog initial incorporation occurs in the myoid of the inner segment which is followed by migration sclerally of a sharp band of radioactivity. The band disappeared within five to seven weeks. From these observations he concluded that a continual renewal of the outer segment, including disk membranes, occurs with synthesis of protein components occurring in the myoid. It is possible that the localization of opsin in the myoid and ellipsoid described here is a reflection of this synthetic and renewal process. 


\section{(d) Comments on a possible extracellular space in outer segments}

Considerable effort has been directed toward determining whether the intradisk space of the rod outer segment disk is continuous with the extracellular compartment (for review see Dowling, 1967). This has involved an analysis of the ultrastructure of aldehyde, osmic acid or permanganate fixed outer segments in adult and immature frogs (Nilsson, 1964a). A consensus of electron microscopists appears to be that the disks lie free within the outer segments and except for those near the foveal end of the outer segment, are not continuous with the external membrane. The situation is clearer for cone outer segments where a number, if not all, of disks along the onter segment appear continuous with the external membrane. The fact that intact rod outer segments on dissected retinas stain following treatment with antirhodopsin serum and fluorescein-labeled sheep anti-rabbit $\gamma$-globulin, is of interest in this regard. It appears that there is a diffusion pathway into the rod outer segment of sufficient size to allow molecular species as large as antibody molecules to enter. Intact outer segments did not appear hollow when viewed end-on after such treatment as would be the case if immune globulin and fluorescein-labeled $\gamma$-globulin were bound only to the external membrane of the rod outer segment. This cannot be confirmed, however, until similar experiments are performed in which the diffusion and binding of ferritin- or peroxidase-labeled antirhodopsin in the retina are examined electron microscopically. The issue of whether an extra-cellular component exists within the rod is critical to models used to explain the mechanism of coupling photon absorption to primary electrical events.

\section{(e) Immunohistochemistry of the pigment epithelium}

The pigment epithelium is characterized morphologically by containing numerous lysosomes, membranous myeloid bodies, and an extensive smooth surfaced endoplasmic reticulum which is apparently continuous, in part, with membranes of the myeloid bodies (Porter \& Yamada, 1960; Dowling, 1967). There is visually a correspondence between structures which stain with Baker's acid hematin reagent and the above structures soon electron microscopically. This is reasonable since the phospholipid content of similar membranous structures is so well documented in other tissues. The structures which stained with Baker's acid hematin, stained with antirhodopsin immune serum. A number of explanations of this finding seem plausible. First, the occurrence of rhodopsin in myeloid bodies has been established microspectrophotometrically (Liebman, Rice, Carroll, Entine \& Laties, 1967). Second, morphologic evidence supports the concept that the continued growth process of the outer segments is coupled with a degradation of outer segment components within the pigment epithelium (Dowling \& Gibbons, 1962; Bairati \& Orzalesi, 1963; Young, 1967). The localization of opsin within pigment epithelium described here is consistent with both these explanations in that the localization within myeloid bodies could be photopigment demonstrable by its characteristic spectral absorption and that in other cytoplasmic components only opsin in a storage or degradative state.

The significant results of this work are as follows.

(1) The development and characterization of a specifie antirhodopsin immune serum.

(2) The demonstration that photopigment occurs uniformly over the surface of disk membranes. 
(3) The localization of rhodopsin antibodies in the myoids and ellipsoids of retinal receptors and in cytoplasmic structures of pigment epithelium including the myeloid bodies indicates a wide distribution of opsin, at least.

(4) The use of the antirhodopsin immune serum has allowed the description of the molecular arrangement of photopigment in retinal receptor disk membranes (Blasie et al., 1969; Blasie \& Worthington, 1969).

This work was supported by United States Public Health Service Grants AM-05197, AM-10084, and NB-07199. We wish to express our appreciation for the guidance and criticism given us during the course of this work by Drs M. Alpern, G. Pierce, and R. Midgely. This work has been reported in part, elsewhere (Dewey, M. M., Davis, P. K. \& Blasie, J. K. (1966). Abstr. The Histochemical Society; Dewey, M. M., Blasie, J. K. \& Davis, P. K. (1967). Abstr. The Biophysical Society).

\section{REFERENCES}

Bairati, A., Jr. \& Orzalesi, N. (1963). J. Ultrastructure Res. 9, 484.

Barr, L. \& Alpern, M. (1963). J. Gen. Physiol. 46, 1249.

Blasie, J. K., Dewey, M. M., Blaurock, A. E. \& Worthington, C. R. (1965). J. Mol. Biol. $14,143$.

Blasie, J. K. \& Worthington, C. R. (1969). J. Mol. Biol. 39, 417.

Blasie, J. K., Worthington, C. R. \& Dewey, M. M. (1969). J. Mol. Biol. 39, 407.

Collins, F. D., Love, R. M. \& Morton, R. A. (1952). Biochem. J. 51, 292.

Dowling, J. E. (1967). In Molecular Organization and Biological Function, ed. by J. M. Allen, p. 186. New York: Harper \& Row.

Dowling, J. E. \& Gibbons, I. R. (1962). J. Cell Biol. 14, 459.

Hubbard, R. (1954). J. Gen. Physiol. 37, 381.

Liebman, P. A., Rice, R., Carroll, S., Entine, G. \& Laties, A. (1967). Invest. Opthal. 6, 214.

Lillie, R. D. (1965). In Histopathologic Technic and Practical Histochemistry, 3rd edn. p. 484. New York: MeGraw-Hill Co.

Nilsson, S. E. G. (1964a). J. Ultrastructure Res. 11, 581.

Nilsson, S. E. G. (1964b). J. Ultrastructure Res. 10, 390.

Porter, K. R. \& Yamada, E. (1960). J. Biophys. Biochem. Cytol. 8, 181.

Wald, G. (1938). J. Gen. Physiol. 21, 795.

Wald, G. (1949). Docum. Opth. 3, 94.

Wald, G. \& Brown, P. K. (1952). J. Gen. Physiol. 35, 797.

Young, R. W. (1967). J. Cell Biol. 33, 61. 